7 Abstract

\title{
Synergetic hydrothermal co-liquefaction of crude glycerol and aspen wood
}

\author{
Thomas H. Pedersen ${ }^{a}$, Lukas Jasiūnas ${ }^{\mathrm{a}}$, Luca Casamassima ${ }^{\mathrm{a}}$, Shashank Singh ${ }^{\mathrm{a}}$, Thomas Jensen ${ }^{\mathrm{b}}$, \\ Lasse A. Rosendahl ${ }^{\text {,** }}$ \\ ${ }^{a}$ Department of Energy Technology, Aalborg University, Pontoppidanstrcede 101, 9220 Aalborg Øst, Denmark \\ ${ }^{b}$ Emmelev A/S, Emmelevgyden 25, 5450 Otterup, Denmark
}

\begin{abstract}
Crude glycerol-assisted hydrothermal co-liquefaction of aspen wood was studied in batch microreactors. An experimental matrix of 14 experiments was defined to investigate the effects of three different process parameters on the yields of biocrude and char, and on biocrude quality. Coprocessing aspen wood and neat glycerol led to a significant reduction in the char yield, and glycerol is hypothesized to act as a radical scavenger, alleviating re-polymerization of especially ligninderived fragments. In the temperature range of $380-420{ }^{\circ} \mathrm{C}$, it was found that biocrude and char yield, and biocrude quality were all invariant to the reaction temperature. By increasing the crude glycerol to aspen wood mass ratio from 0:1 to 3:1, char yield was decreased from $18.3 \%$ (only aspen wood) to $3.4 \%$. Furthermore, the biocrude quality in terms of the effective hydrogen-tocarbon ratio $\left(\mathrm{H} / \mathrm{C}_{\mathrm{eff}}\right)$ was significantly affected by the crude glycerol to aspen wood ratio. At a crude glycerol to aspen wood ratio of $3: 1$ the $\mathrm{H} / \mathrm{C}_{\text {eff }}$ was 1.6 compared to 0.96 in the case of only aspen wood. The chemical composition of the biocrude was observed to be directly influenced by the two main feedstock constituents, which allows straightforward composition prediction of the biocrude, and hence enables controllability of the biocrude properties by feedstock mixing.

Keywords: Biocrude, Biofuel, Biomass, Resource management, Hydrothermal liquefaction, Bio-refinery
\end{abstract}

\section{Introduction}

Hydrothermal liquefaction of lignocellulosic biomass is an energy- and resource-efficient chemical route for renewable production of oxygenated precursors for hydrocarbon fuels in the form of a biocrude. Optimization of the process chemistry involves an efficient breakdown of biomass

${ }^{*}$ Corresponding author

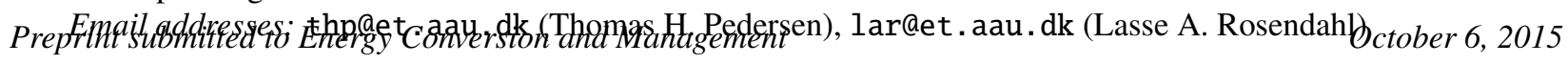


macromolecules (cellulose, hemicellulose and lignin) to low molecular weight intermediates, and subsequent synthesis of these intermediates for maximum yields of value-added deoxygenated compounds. Lignin, in particular, is a valuable macromolecule for production of oxygenated aromatics, mainly in the form of phenolics. However, lignin shows strong tendencies to char formation through thermally induced radical pathways when processed under severe processing conditions. Radicals, which ultimately lead to retrogressive repolymerization reactions, cause the formation of inactive high molecular weight char and thus limits the yield of liquid compounds. However, lignin-derived radicals can be stabilized by the addition of a radical scavenger preventing char formation and enhancing the yield of liquid products. Wood liquefaction in near-critical water and co-solvents, primarily alcohols inspired by organosolv pulping, has shown the ability to stabilize lignin radicals $[1,2]$. Demirbas et al. also investigated wood liquefaction in anhydrous glycerol with and without the presence of an alkaline catalyst [3-5]. It was found that in the presence of an alkaline catalyst wood can be completely liquefied in glycerol.

Glycerol, a bio-derived polyalcohol, is produced mainly as a byproduct from the biodiesel industry and is a commodity chemical used in a broad variety of commercial applications [6]. Crude glycerol, the unrefined byproduct from the transesterification step in biodiesel production, is a bulk emulsion of mainly glycerol, salts, and transesterification residues, such as free fatty acids (FFAs) and trapped fatty acid methyl esters (FAMEs), the main chemical compounds of biodiesel. Ever since the market entry of biodiesel, the glycerol market has been altered dramatically and in an increasing biodiesel production scenario, a glycerol market saturation is likely to occur. Therefore, the market value of glycerol and especially crude glycerol is in a decreasing trend [7]. The decreasing market value offers alternative chemistry and energy opportunities [8-10].

Wolfson et al. investigated the uses of glycerol and crude glycerol as a green solvent and reaction medium for various chemical reactions [11, 12]. Base-catalyzed reactions such as Aldol condensation and redox reactions, where glycerol is utilized as a hydrogen donor, were researched. In the latter case, glycerol oxidizes to dihydroxyacetone by donation of hydrogen. In a biomass-toliquid (BtL) fuel context through hydrothermal liquefaction, glycerol has also shown enhancing effects on the liquid phase product when mixed and processed with other biomass feedstock [13-17]. Ye et al. investigated co-liquefaction of swine manure and crude glycerol in a model compound study to investigate reaction pathways. It was concluded that all constituents of crude glycerol 
(glycerol, methanol, fatty acids) showed positive effects and enhanced the liquid yield. Esterification of methanol and fatty acids as well as acid catalyzed dehydration reactions for aromatics formation were mentioned as possible pathways.

In supercritical water, glycerol undergoes multiple and complex chemical transformations, but most interesting in the BtL context are the radical formation and hydrogen transfer reactions $[6,8,11,12]$. It is hypothesized that these hydrogen transfer reactions can be governing for stabilizing lignin-derived radicals when processed with lignocellulosic materials and thus a potential scavenging chemical agent leading to substantial char reduction.

The main objective of this study is to experimentally investigate the synergetic effects of coliquefaction of crude glycerol and aspen wood at supercritical water conditions, and to attempt to set up a reaction framework for the conversion process. The study aims to examine the effects of process conditions and feedstock composition on the product yields and product quality. Furthermore, the study aims to elucidate the impact of the bio-feedstock compounds (cellulose, hemicellulose, lignin, glycerol, and lipids) on the process chemistry as well as on the fuel characteristics of the resulting biocrudes.

\section{Materials and Methods}

The crude glycerol used in this study was acquired from a Danish biodiesel producer (Emmelev A/S). The crude glycerol was collected right after the esterification process and therefore strongly alkaline due to the presence of residual biodiesel catalyst. A small sample of the crude glycerol was analyzed in-house and the characteristics are compiled in Table 1. These should not be interpreted as generally representing the common crude glycerol composition from that producer, but rather as a single small sample analyzed for the purpose of this work. In order to estimate the amount of fatty acid methyl esters (FAMEs) and unconverted fatty acids (FFAs) in the crude glycerol, the crude glycerol was mixed and stirred with a HCL-solution, breaking the crude glycerol emulsion and causing a phase separation. The FFAs and FAMEs were extracted by adding hexane to the total mixture. The total mixture was then decanted to obtain the supernatant phase comprising hexane, FFAs, and FAMEs. Hexane was removed from the hexane fraction by vacuum rotary evaporation at $40{ }^{\circ} \mathrm{C}$. The total amount of FFAs and FAMEs is presented in Table 1. Neat glycerol (99.5\%) was purchased from Brenntag Nordic A/S. Calorimetry of this fraction revealed a higher heating value 
87 of 38.9 MJ and GC-MS analysis showed a mixture of FAMEs, FFAs and trace amounts of glycerol.

Table 1: Aspen wood and crude glycerol analyses based on dry, ash-free basis. The moisture content of the aspen wood was $3.6 \%$.

\begin{tabular}{lcc}
\hline & Aspen wood & Crude glycerol \\
\hline Elemental analysis [wt. \% ${ }^{a}$ & & \\
$\mathrm{C}$ & 50.39 & 52.08 \\
$\mathrm{H}$ & 6.19 & 11.59 \\
$\mathrm{O}$ & 43.23 & 36.33 \\
$\mathrm{~N}$ & 0.19 & - \\
HHV [MJ/kg] & 18.75 & 25.51 \\
Ash [wt. \%] ${ }^{b}$ & 0.46 & 4.06 \\
Cellulose [wt. \%] & 47.14 & - \\
Hemicellulose [wt. \%] & 19.64 & - \\
Lignin [wt. \%] & 22.11 & - \\
FFAs + FAMEs [wt. \%] & - & 45.53 \\
Glycerol [wt. \%] ${ }^{c}$ & - & 49.51 \\
Methanol [wt. \%] & - & 0.37 \\
Water [wt. \%] $^{a}$ oxygen calculated by difference & & \\
${ }^{b}$ ash measured by heating a sample to $800{ }^{\circ} \mathrm{C}$ for one hour \\
${ }^{c}$ calculated by difference & & \\
\hline
\end{tabular}

\subsection{Experimental procedure}

All experiments were carried out in stainless steel (316) micro reactors, each reactor having an operational volume of $10 \mathrm{~mL}$. In total, 14 independent experiments were conducted in triplicates. The effects of feedstock composition (neat/crude glycerol to aspen wood mass ratio), feedstock 
mass loading of the total slurry mass and the reaction temperature were investigated. The feedstock composition was ranged from only neat and crude glycerol to only aspen wood. Three total feedstock mass loadings of 10, 20 and $30 \%$ were examined, and three reaction temperature levels, 380,400 and $420^{\circ} \mathrm{C}$.

In each of the runs, aspen wood was mixed with neat or crude glycerol and distilled water to form the predefined feedstock slurry. A total of $5 \mathrm{~g}$ of slurry was then loaded into the reactors. High pressure nitrogen gas was used to simultaneously pressure test ( 80 bar) and purge the reactors to evacuate oxygen. Hereafter, three reactors were mechanically coupled and, together with a thermocouple, mounted to an agitator providing mechanical mixing of the reagents inside the reactors while processed. The three reactors were submerged into a preheated, fluidized sand bath and held for 15 minutes of retention time under autogenous reaction pressure. As baseline, neat glycerol and aspen wood at different mass ratios were co-processed in an alkaline aqueous medium. For these experiments neat glycerol and aspen were mixed in distilled water at a fixed feedstock mass loading of $20 \%$ of the total slurry mass. $\mathrm{K}_{2} \mathrm{CO}_{3}$ was added as base catalyst to mimic the alkaline nature of the crude glycerol. The mass of added $\mathrm{K}_{2} \mathrm{CO}_{3}$ amounted to $10 \%$ of the total mass of neat glycerol and aspen wood.

After 15 minutes of retention time, the reactors were cooled by quenching in water and the gas phase products were vented from the reactors through top mounted valves. The remaining products in the reactors consisted of a solid residue, referred to as char, a biocrude and an aqueous phase. The reactors were washed with acetone in order to obtain a homogeneous liquid phase and char. The liquid phase was separated from the char by vacuum assisted mechanical filtration. Char, defined as the water and acetone-insoluble solid fraction was then obtained by weighing the solid residue after drying for at least 24 hours at $105^{\circ} \mathrm{C}$. Acetone was evaporated from the homogeneous liquid fraction and the remaining liquid fraction was extracted using diethyl ether. The ether-soluble extracts were defined as biocrude. Char and biocrude yields were calculated on a dry, ash-free (daf) basis according to Eq. 1. Experimental error was within $5 \%$.

$$
\text { Yield }_{\text {phase }}=\frac{\text { Weight of phase }}{\text { Weight of dry, ash-free feedstock }}
$$

Elemental analysis was carried out on Perkin Elmer 2400 II Series. For each of the obtained biocrudes, an effective hydrogen-to-carbon ratio $\left(\mathrm{H} / \mathrm{C}_{\mathrm{eff}}\right)$ was calculated according to Eq. 2 . 


$$
H / C_{e f f}=\frac{H-2 O}{C}
$$

GC-MS analyses of all biocrude samples were carried out on a Thermo Scientific Trace 1300 ISQ GC/MS system (Length: 30 m., i.d.: 0.25 mm., um: 0.25 um um, TG-SQC column). All samples were derivatized using a BSTFA reagent prior to the analysis. Samples were then diluted with diethyl ether (DEE) and subjected to the following oven temperature profile; ramped from 40 ${ }^{\circ} \mathrm{C}$ to $300{ }^{\circ} \mathrm{C}$ at $10{ }^{\circ} \mathrm{C} / \mathrm{min}$. Injector and ion source temperatures were $300{ }^{\circ} \mathrm{C}$, split ratio was 1:20, and flow rate of the carrier gas (helium) was $1.0 \mathrm{~mL} / \mathrm{min}$.

\section{Results and Discussion}

A total of 14 experiments were conducted to assess processing aspects and synergies when co-liquefying crude glycerol and aspen wood into a biocrude. Table 2 summarizes the nine experiments conducted with crude glycerol and presents the processing parameters in the individual experiments. The biocrude and char yield results obtained from the neat glycerol and aspen wood experiments are presented in Figure 2. In terms of mass ratio between neat or crude glycerol and aspen wood, the composition was varied between the two extremes, pure crude glycerol and pure aspen wood. In the extreme of pure crude glycerol (Run \#1), it is observed that the emulsified appearance of the original crude glycerol remained even after processing. In all other experiments, two distinct phases were observed, an aqueous phase and a biocrude phase. It is believed that low molecular weight carboxylic acids, produced from aspen wood degradation, positively break the crude glycerol emulsion comprised of saponified compounds of FFAs base catalyst. FFAs are then clearly separated from the aqueous phase and add value to the resultant biocrude phase.

\subsection{Biocrude composition}

From the experiments conducted it was found that mainly three classes of compounds were obtained in the biocrudes; 1) oxygenates derived from glycerol and aspen wood degradation, 2) unconverted FFAs and, 3) FAMEs which were transferred almost intact from the crude glycerol to the biocrude phase. A similar observation was done by Leow et al. by processing micro algae at subcritical water conditions [18]. Unconverted glycerol was also found in the biocrudes. Figure 1 
Table 2: Summary of the experiments conducted. The table includes the experimental parameters in each experiments together with the yields of biocrude and char, and the $\mathrm{H} / \mathrm{C}_{\text {eff }}$ ratios of the resulting biocrudes.

\begin{tabular}{lccccccccc}
\hline \multicolumn{10}{c}{ Experimental runs: } \\
\hline Conditions: & $\mathbf{1}$ & $\mathbf{2}$ & $\mathbf{3}$ & $\mathbf{4}$ & $\mathbf{5}$ & $\mathbf{6}$ & $\mathbf{7}$ & $\mathbf{8}$ & $\mathbf{9}$ \\
CG-Wood ratio & $1: 0$ & $3: 1$ & $1: 1$ & $1: 1$ & $1: 1$ & $1: 1$ & $1: 1$ & $1: 3$ & $0: 1$ \\
Mass loading [wt.\%] & 20 & 20 & 10 & 20 & 20 & 20 & 30 & 20 & 20 \\
Temperature [ ${ }^{\circ} \mathrm{C}$ ] & 400 & 400 & 400 & 380 & 400 & 420 & 400 & 400 & 400 \\
Results: & & & & & & & & & \\
Biocrude yield [wt.\%] & - & 48.74 & 50.97 & 38.05 & 40.71 & 41.60 & 43.35 & 52.26 & 42.3 \\
Char yield [wt.\%] & - & 3.42 & 3.65 & 3.59 & 5.16 & 4.29 & 7.78 & 7.41 & 18.30 \\
H/C eff & - & 1.63 & 1.57 & 1.45 & 1.44 & 1.43 & 1.44 & 1.19 & 0.96 \\
\hline
\end{tabular}

shows a chromatogram of experimental Run \#5, depicting all three classes of compounds. It is observed that the volatile fraction of aspen wood-derived compounds is a complex mixture, of which many of the constituents were unidentifiable, but showed ketones e.g. and oxygenated aromatics having number of carbon atoms mainly in the range of $\mathrm{C}_{6}-\mathrm{C}_{10}$. Ketones are hypothesized to be formed by condensation reactions of carbohydrate-derived intermediates. Oxygenated aromatics are mainly products of lignin degradation. Palmitic and oleic acid methyl esters were the only observed FAMEs. These correspond to their FFAs equivalents, namely palmitic and oleic acids. Table 3 lists the most abundant compounds (the GC-MS peak area) observed in experimental Run \#5.

\subsection{Effects of processing glycerol and wood}

From Figure 2 it readily appears that by mixing aspen wood with glycerol char formation can be significantly reduced. Starting at a char yield of approximately $18 \%$ in the case of only aspen wood, the yield of char decreases almost exponentially to a char yield of $2.7 \%$, when processing only glycerol. The fact that the char yield does not follow a linear trend indicates that the char reduction by co-processing glycerol and aspen wood is not only a mixing effect, but also a chemical interaction effect between the feed constituents. Moreover, it is observed that when processing only glycerol, a biocrude is also obtained. The mass fraction of biocrude obtained was determined to $17.7 \%$ that mainly consisted of ketones and aromatics. The observation that biocrude and char is 
Table 3: Main chemical compounds obtained from the biocrude from experimental run \#5. The number of carbon atoms, $\mathrm{C \#}$, of the compounds is also presented.

\begin{tabular}{llcc}
\hline RT (min) & \multicolumn{1}{c}{ Identified compound } & Chemical Formula & C\# \\
\hline 6.25 & 1,2-propanediol & $\mathrm{C}_{3} \mathrm{H}_{8} \mathrm{O}_{2}$ & 3 \\
6.28 & 2-cyclopenten-1-one, 2,3-dimethyl- & $\mathrm{C}_{7} \mathrm{H}_{10} \mathrm{O}$ & 7 \\
7.01 & Phenol & $\mathrm{C}_{6} \mathrm{H}_{6} \mathrm{O}$ & 6 \\
7.30 & Hexanoic acid & $\mathrm{C}_{6} \mathrm{H}_{12} \mathrm{O}_{2}$ & 6 \\
8.20 & Phenol, 2-methyl- & $\mathrm{C}_{7} \mathrm{H}_{8} \mathrm{O}$ & 7 \\
8.60 & Cyclohexanecarboxylic acid & $\mathrm{C}_{7} \mathrm{H}_{12} \mathrm{O}_{2}$ & 7 \\
8.75 & Heptanoic acid & $\mathrm{C}_{7} \mathrm{H}_{14} \mathrm{O}_{2}$ & 7 \\
9.07 & 1-Penten-3-ol, 4-methyl- & $\mathrm{C}_{6} \mathrm{H}_{12} \mathrm{O}$ & 6 \\
10.11 & Octanoic acid & $\mathrm{C}_{8} \mathrm{H}_{16} \mathrm{O}_{2}$ & 8 \\
10.38 & Glycerol & $\mathrm{C}_{3} \mathrm{H}_{8} \mathrm{O}_{3}$ & 3 \\
10.94 & Catechol & $\mathrm{C}_{6} \mathrm{H}_{6} \mathrm{O}_{2}$ & 6 \\
11.92 & 4-methylcatechol & $\mathrm{C}_{7} \mathrm{H}_{8} \mathrm{O}_{2}$ & 7 \\
12.83 & 2-hydroxyphenyl ethanol & $\mathrm{C}_{8} \mathrm{H}_{10} \mathrm{O}_{2}$ & 8 \\
13.8 & 4-hydroxyphenylacetic acid & $\mathrm{C}_{8} \mathrm{H}_{8} \mathrm{O}_{3}$ & 8 \\
17.9 & Palmitic acid, methyl ester & $\mathrm{C}_{17} \mathrm{H}_{34} \mathrm{O}_{2}$ & 17 \\
19.08 & Palmitic acid & $\mathrm{C}_{16} \mathrm{H}_{32} \mathrm{O}_{2}$ & 16 \\
19.59 & Oleic acid, methyl ester & $\mathrm{C}_{19} \mathrm{H}_{36} \mathrm{O}_{2}$ & 19 \\
20.65 & Oleic acid & $\mathrm{C}_{18} \mathrm{H}_{34} \mathrm{O}_{2}$ & 18 \\
\hline & & & \\
\hline
\end{tabular}

obtained when processing glycerol points towards the fact that condensation reactions of glycerol or compounds derived from glycerol decomposition occur. Hence, glycerol does not only act as char-reducing agent, but also contributes to the biocrude formation. The interaction effects are also observed from the non-linear trend in the biocrude yield for varying mass ratios of glycerol and aspen wood. Pure mixing effects would have resulted in a straight line between the extreme points (only aspen wood, and only glycerol). However, when adding small amounts of glycerol to aspen wood, the biocrude yield was acually slightly increasing from approximately $42 \%$ to approximately $45 \%$, comfirming the occurence of interaction reactions. For further increase in the glycerol fraction in the feedstock mixture, the char yield decreases almost linearly indicating pure mixing effects before it levels out around $2.7 \%$ as it approaches the feed composition of pure glycerol.

Apart from condensation reactions of glycerol leading to ether-soluble compounds, it is hypothesized that glycerol also acts as a lignin radical scavenger via alkaline-assisted hydrogen abstraction/donation reactions. Figure 3 shows a proposed reaction scheme for glycerol degradation reactions. Under alkaline conditions, glycerol has previously been observed to act as a hydrogen 
donor, where it undergoes dehydrogenation reactions leading to the formation of dihydroxyacetone [12]. Donor-hydrogen may then stabilize radicals, reducing or saturating reactive compounds and thereby reducing the amount of char. Thermally induced degradation of glycerol may also lead to the formation of ethanol and formic acid via $\mathrm{C}-\mathrm{C}$ bond cleavage. Ethanol and formic acid have previously been used as radical scavengers and hydrogen donors for minimizing char formation during wood and lignin liquefaction $[1,19,20]$. Another source of hydrogen donors may be gasification of intermediate compounds. Glycerol may dehydrate into hydroxyacetone and then thermally degrade into formaldehyde and acetaldehyde; intermediates for methane, carbon dioxide, carbon monooxide, and hydrogen production [21].

\subsection{Effects of reaction temperature}

The effects of processing temperature on the yield and quality of the biocrudes were investigated at constant feed mixture composition. From Table 2 (Run \#4, \#5, and \#6), it can be seen that neither the biocrude nor the char yields or even the biocrude quality are strongly affected by varying the reaction temperature. There is a slight tendency of increasing biocrude yield as a function of increasing temperature, but this is insignificant taking experimental variations into account. From GG-MS analysis identical compounds were found throughout the temperature variations, which is also reflected in the constant $\mathrm{H} / \mathrm{C}_{\text {eff }}$ measurements. Fatty acids have previously been proven to be thermally stable in subcritical water, although isomerization has been observed [18, 22, 23]. The present results show that saturated and mono-unsaturated fatty acids are also partially stable at supercritical water conditions.

\subsection{Effects offeedstock mass loading}

The effects of feedstock mass loading on the yield and quality of the biocrudes were also investigated (Run \#3, \#5, and \#7). Increasing the feedstock mass loading from 10 to $20 \%$ gives lower biocrude yields. A further increase in mass loading to $30 \%$ indicates an increase in biocrude yield, but this is not statistically significant but within experimental error. Contrary, the char yields are observed to increase almost linearly with increasing feedstock mass loadings. whereas a high crude glycerol to aspen wood ratio and a low feed mass fraction had a positive affect on the liquefaction process in the form of a lowered yield of chars. Since the char formation from crude glycerol is significantly smaller than the char formation from aspen wood, it is believed that the decrease in 
the char yield, when lowering the mass loading is an aspen wood concentration effect. As the mass loading decreases the fragments from aspen wood decomposition are more dispersed in the aqueous solution, which decreases the tendency to repolymerize. No significant change in $H / C_{\text {eff }}$ was observed while altering the mass loading.

\subsection{Effects of crude glycerol to aspen mass ratio}

Changing the mass ratios between crude glycerol and aspen wood led to substantial changes both in terms of yields and quality. As in the case of neat glycerol and aspen, Figure 2 shows a similar trend in that the char is substantially reduced when pure aspen wood is mixed with crude glycerol. The biocrude and char yields from the neat glycerol and aspen wood experiments are overlaid for comparison. Again, starting at a char yield of approximately $18 \%$ in the case of only aspen wood, the yield of char decreases almost exponentially to a char yield less than $4 \%$, close to that of neat glycerol and aspen wood (2.7\%). When increasing the mass fraction of crude glycerol to aspen wood beyond 1:3, the reduction in char formation is only marginal, and the char yield seems more linear in this range. This observation could indicate that the further reduction in char formation is more likely mixing effects rather than chemical interactions. Furthermore, the observation, that the char yields are almost equal whether aspen wood is co-processed with neat or crude glycerol, dictates that FFAs and FAMEs do not, or only marginally, contribute to char formation and reduction.

Moreover, when co-processing even small amounts of crude glycerol with aspen wood, the biocrude yield was also observed to increase, which is explained by the glycerol and aspen wood chemical interaction. It is hypothesized that the increase in the biocrude yield was a direct effect of the suppression of char forming reactions, especially from the lignin fraction of the aspen wood. Stabilization of retrogressive reactions between lignin fragments during hydrothermal degradation reduced the char formation through formation of low molecular weight compounds, which then contributed to a higher biocrude yield. As the crude glycerol to aspen wood ratio increases, it is assumed that no further stabilization reactions occur which could increase the biocrude yield. Until a 1:1 ratio of aspen wood and crude glycerol, the biocrude mass yield follows the trend for neat glycerol and aspen wood but is offset due the contribution of the FFAs and FAMEs fraction, which transfer almost unconverted to the biocrude. As the crude glycerol fraction increases so does the amount of FFAs and FAMEs, and thus the increase in biocrude yields can be explained by the 


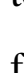
feedstock, aspen wood becomes the primary of the biocrude, and vice versa. In terms of $\mathrm{H} / \mathrm{C}_{\text {eff }}$, the compounds derived from aspen wood are generally of a lower quality as compared to FAMEs and FFAs. This is observed in Figure 2, where a linear relation between the $\mathrm{H} / \mathrm{C}_{\text {eff }}$ and the crude glycerol to aspen wood ratio is shown. At pure aspen wood conditions, the $\mathrm{H} / \mathrm{C}_{\text {eff }}$ is below unity, and then increases to a $\mathrm{H} / \mathrm{C}_{\text {eff }}$ above 1.6, resembling FAMEs and FFA, as crude glycerol becomes the greater part of the feedstock. The linear trend observed for the biocrude quality suggests that the chemical interaction between the feed constituents, resulting in the nonlinear yield trends, does not alter the quality and is thus purely effects of mixing. The trend that FFAs and FAMEs become the dominant compounds of the biocrude at high crude glycerol to aspen wood ratios is depicted in Figure 4. Here it is shown that the relative abundance (by peak area) of aspen wood-derived compounds increases for larger shares of aspen wood in the feed. The shift in biocrude compounds abundance is a desirable asset of the process, allowing the resulting biocrude to be tuned for intended purposes by changing the feedstock composition. Another interesting observation is that at a high share of aspen wood, FAMEs are absent from the biocrude. It is believed that the disappearance of FAMEs is caused by acid catalyzed hydrolysis reactions. Such reaction is given in Eq. 3, where palmitic acid methyl ester is hydrolyzed into palmitic acid and methanol.

$$
\mathrm{C}_{17} \mathrm{H}_{34} \mathrm{O}_{2}+\mathrm{H}_{2} \mathrm{O} \underset{\text { hydrolysis }}{\stackrel{\text { Acid catalyzed }}{\longrightarrow}} \mathrm{C}_{16} \mathrm{H}_{32} \mathrm{O}_{2}+\mathrm{CH}_{3} \mathrm{OH}
$$

As mentioned, when aspen wood decomposes carboxylic acids such as acetic acid and propionic acid are produced. These carboxylic acids are responsible for breaking the crude glycerol emulsion, but they also change the initial alkaline feedstock into an acidic product. Since the crude glycerol is the carrier of the alkaline base, and that aspen wood is the main contributor to acids formation, 
the lower the crude glycerol to aspen wood ratio, the less basic the product gets. For example, in Run \#2 (3:1 ratio) and \#8 (1:3 ratio), the $\mathrm{pH}$ of the feedstock was 11.57 and 9.48, respectively. The higher $\mathrm{pH}$ in Run \#2 is the natural cause of a higher share of crude glycerol. The corresponding $\mathrm{pH}$ values of the aqueous products of Run \#2 and \#8 were 6.28 and 5.00, respectively. Hydrolysis of FAMEs to their corresponding FFAs is obviously an undesirable reaction, since the conversion of FFAs into FAMEs in the first place provides a means for enhancing biodiesel properties. For low aspen wood fractions in the feedstock it therefore appears that the FAMEs and FFAs proceed through the process unchanged. In this case the amount of FAMEs and FFAs in the biocrude can then be easily predicted by knowing the amount of these compounds in the feedstock.

\section{Conclusions}

Synergies of biocrude production from crude glycerol and aspen wood co-liquefaction were investigated. The composition of all biocrudes was found to be dominated by oxygenates from wood degradation, unconverted FFAs and FAMEs brought by the crude glycerol. Wood-derived organic acids provided a benign recovery of FFAs and FAMEs in the crude glycerol, by breaking the saponified emulsion. FFAs passed through the process unchanged, whereas FAMEs were hydrolyzed into corresponding FFAs at high aspen wood shares in the feedstock. Biocrude and char yield, biocrude composition and quality were found invariant to reaction temperature. Similarly, the $\mathrm{H} / \mathrm{C}_{\text {eff }}$ ratio was found invariant to the feedstock mass loadings. However, higher mass loadings lead to lower biocrude yields. Altering the crude glycerol and aspen wood ratio lead to significant changes in the resultant biocrude. Char formation was reduced significantly (from $18 \%$ to $<5$ $\%$ ), whereas an increase in biocrude yield was observed. Furthermore, the quality of the product improved in terms of its $\mathrm{H} / \mathrm{C}_{\text {eff }}$ ratio (from $<1$ to $>1.6$ ). The observed direct influence of the two main feedstock constituents, crude glycerol and aspen wood, enables straightforward composition prediction of the product. The synergic utilization of crude glycerol from biodiesel production for further biofuel production is an interesting concept in a bio-refinery context.

\section{Acknowledgements}

This work is part of the Flexifuel Project, a Sino-Danish collaboration, and C3BO (Center for BioOil) at the Department of Energy Technology, Aalborg University. The research was financially 
supported by The Danish Agency for Science, Technology and Innovation (grant no. 10-094552) and The Danish Council for Strategic Research (grant no. 1305-00030B).

\section{References}

[1] Cheng S, D'cruz I, Wang M, Leitch M, Xu CC. Highly efficient liquefaction of woody biomass in hot-compressed alcohol-water co-solvents. Energy \& Fuels 2010;24(9):4659-67.

[2] Hoffmann J. Pedersen T, Rosendahl L. Near-critical and supercritical water and their applications for biorefineries; chap. Hydrothermal Conversion in Near-Critical Water A Sustainable Way of Producing Renewable Fuels. Springer Netherlands. ISBN 978-94-017-8922-6; 2008, p. 373-400. doi:10.1007/978-94-017-8923-3.

[3] Demirbas A. Conversion of biomass using glycerin to liquid fuel for blending gasoline as alternative engine fuel. Energy Conversion and Management 2000;41(16):1741 -8. URL: http: //wwW. sciencedirect.com/science/article/pii/S0196890400000157. doi:http://dx.doi.org/10.1016/S0196-8904(00)00015-7.

[4] Demirbas A. Mechanisms of thermal degradation of wood in alkaline glycerol. Energy Sources, Part A: Recovery, Utilization, and Environmental Effects 2009;31(14):1294-9.

[5] Demirbas A. Biofuels from beech wood via thermochemicals conversion methods. Energy Sources, Part A: Recovery, Utilization, and Environmental Effects 2009;32(4):346-54.

[6] Bagheri S, Julkapli NM, Yehye WA. Catalytic conversion of biodiesel derived raw glycerol to value added products. Renewable and Sustainable Energy Reviews 2015;41(0):113 -27.

[7] Quispe CAG, Coronado CJR, Jr. JAC. Glycerol: Production, consumption, prices, characterization and new trends in combustion. Renewable and Sustainable Energy Reviews $2013 ; 27(0): 475-93$.

[8] May A, Salvadó J, Torras C, Montané D. Catalytic gasification of glycerol in supercritical water. Chemical Engineering Journal 2010;160(2):751 -9. 
[9] Leoneti AB, Aragão-Leoneti V, de Oliveira SVWB. Glycerol as a by-product of biodiesel production in brazil: Alternatives for the use of unrefined glycerol. Renewable Energy 2012;45(0):138-45.

[10] Yang F, Hanna MA, Sun R. Value-added uses for crude glycerol-a byproduct of biodiesel production. Biotechnology for Biofuels 2012;5(1):13.

[11] Wolfson A, Litvak G, Dlugy C, Shotland Y, Tavor D. Employing crude glycerol from biodiesel production as an alternative green reaction medium. Industrial Crops and Products 2009;30(1):78-81.

[12] Wolfson A, Dlugy C, Shotland Y, Tavor D. Glycerol as solvent and hydrogen donor in transfer hydrogenation-dehydrogenation reactions. Tetrahedron Letters 2009;50(43):5951 -3.

[13] Jin Y, Ruan X, Cheng X, Lü Q. Liquefaction of lignin by polyethyleneglycol and glycerol. Bioresource Technology 2011;102(3):3581 -3.

[14] Xiu S, Shahbazi A, Shirley V, Mims MR, Wallace CW. Effectiveness and mechanisms of crude glycerol on the biofuel production from swine manure through hydrothermal pyrolysis. Journal of Analytical and Applied Pyrolysis 2010;87(2):194 -8.

[15] Xiu S, Shahbazi A, Shirley VB, Wang L. Swine manure/crude glycerol co-liquefaction: Physical properties and chemical analysis of bio-oil product. Bioresource Technology 2011;102(2):1928-32.

[16] Xiu S, Shahbazi A, Wallace CW, Wang L, Cheng D. Enhanced bio-oil production from swine manure co-liquefaction with crude glycerol. Energy Conversion and Management 2011;52(2):1004-9.

[17] Ye Z, Xiu S, Shahbazi A, Zhu S. Co-liquefaction of swine manure and crude glycerol to bio-oil: Model compound studies and reaction pathways. Bioresource Technology 2012;104(0):783-7.

[18] Leow S, Witter JR, Vardon DR, Sharma BK, Guest JS, Strathmann TJ. Prediction of microalgae hydrothermal liquefaction products from feedstock biochemical composition. Green Chem 2015;17:3584-99. doi:10.1039/C5GC00574D. 
[19] Kleinert M, Gasson JR, Barth T. Optimizing solvolysis conditions for integrated depolymerisation and hydrodeoxygenation of lignin to produce liquid biofuel. Journal of Analytical and Applied Pyrolysis 2009;85(12):108 -17.

[20] Onwudili JA, Williams PT. Catalytic depolymerization of alkali lignin in subcritical water: influence of formic acid and $\mathrm{pd} / \mathrm{c}$ catalyst on the yields of liquid monomeric aromatic products. Green Chem 2014;16:4740-8. doi:10.1039/C4GC00854E.

[21] Knezevic D. Hydrothermal conversion of biomass. Ph.D. thesis; Enschede; 2009. URL: http://doc. utwente.nl/67359/.

[22] W. King J, L. Holliday R, R. List G. Hydrolysis of soybean oil . in a subcritical water flow reactor. Green Chem 1999;1:261-4. doi:10.1039/A908861J.

[23] Holliday RL, King JW, List GR. Hydrolysis of vegetable oils in sub- and supercritical water. Industrial \& Engineering Chemistry Research 1997;36(3):932-5. doi:10.1021/ie960668f. 


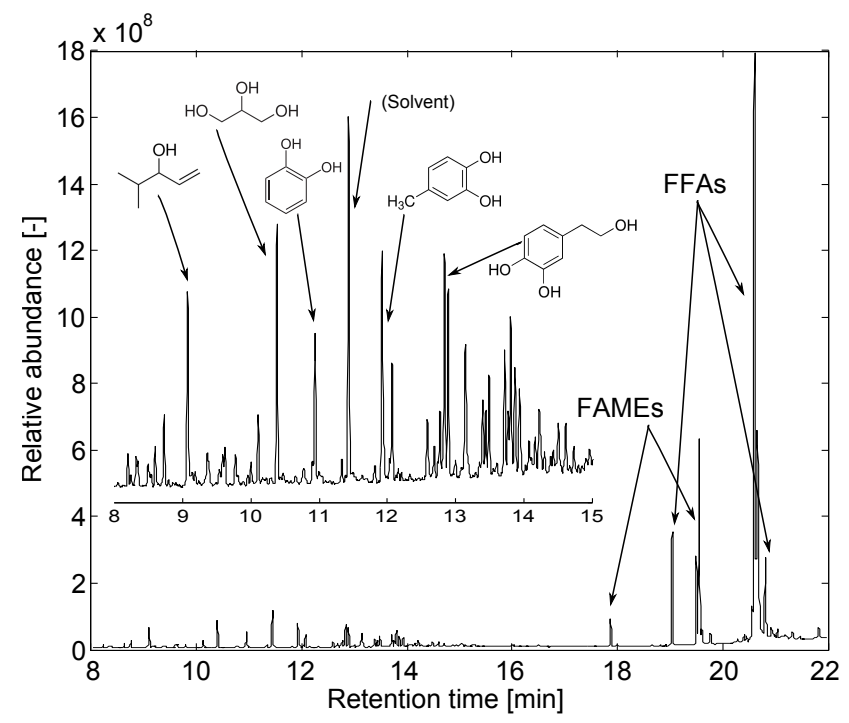

Figure 1: Chromatographic presentation of the main compounds observed in the biocrude from experimental Run \#5. Retention time from 8-15 minutes is enlarged.
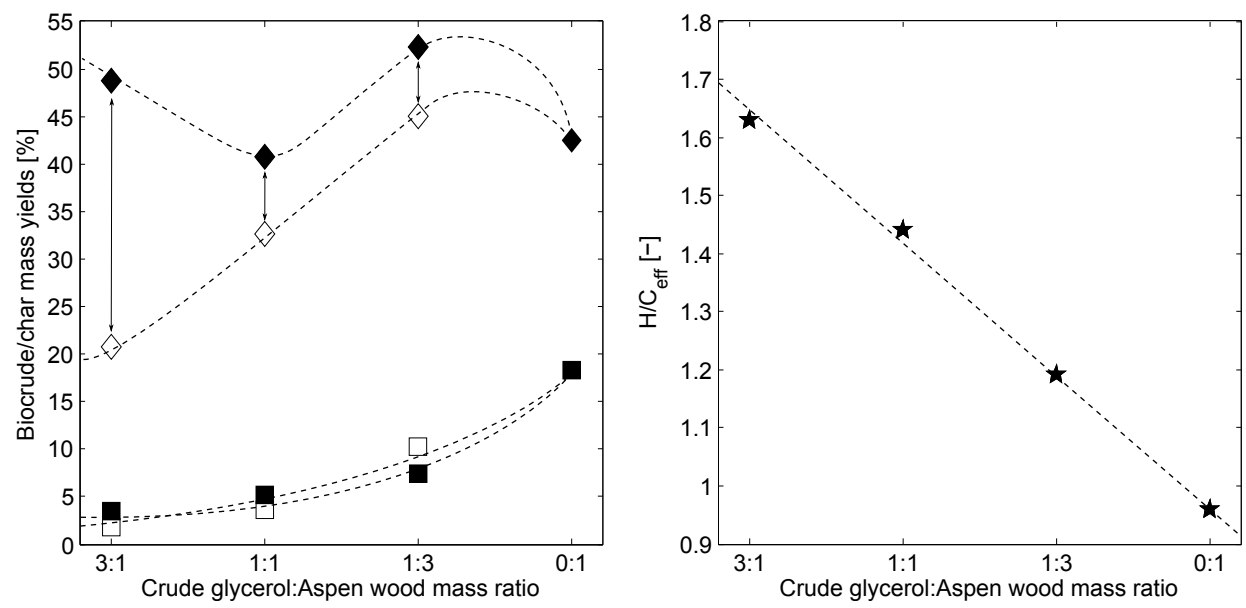

Figure 2: Left: Biocrude ( $\bullet$ and char $(\boldsymbol{\square})$ yields as a function of crude glycerol to aspen wood mass ratio in the feedstock. Overlay of biocrude $(\diamond)$ and char $(\square)$ yields from Figure 2 for comparison between neat and crude glycerol co-processing with aspen wood. Right: $\mathrm{H} / \mathrm{C}_{\text {eff }}(\star)$ as a function of crude glycerol to aspen wood mass ratio in the feedstock. 


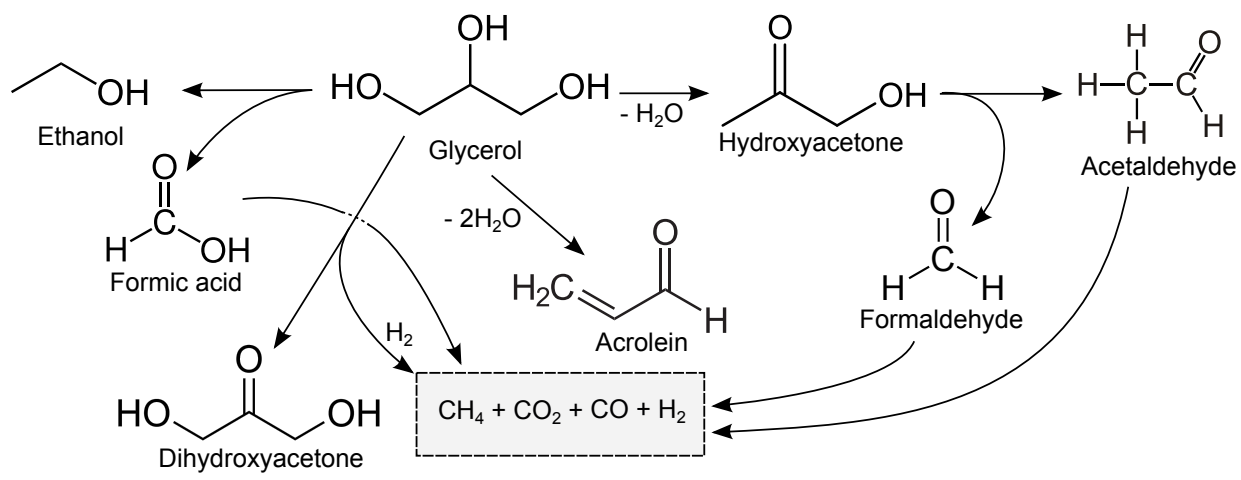

Figure 3: Proposed reaction scheme for degradation reactions of glycerol in supercritical water.

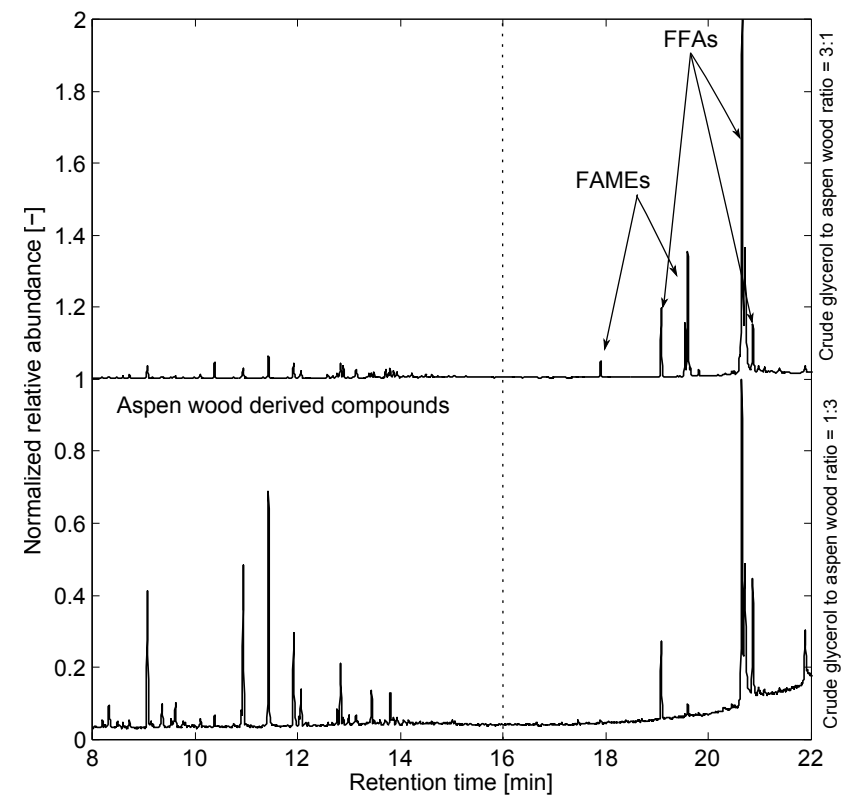

Figure 4: Normalized chromatograms of experimental Run \#2 (top) and Run \#8 (bottom). 